\title{
The positioning errors detection and compensation of translational axes for five-axis measuring system based on VDI-3441 criterion
}

\author{
Fei Zhigen ${ }^{a}$,Yang Wenjian ${ }^{b}$ \\ Mechanical \& Electrical Engineering Department Zhengzhou University of Light Industry, \\ Zhengzhou 45002, China \\ afzg2006@stu.xjtu.edu.cn, bwenjianhust@163.com
}

Keywords: Five-axis measuring system, Error compensation of translational axes, VDI-3441 criterion

\begin{abstract}
In order to improve the positioning accuracy of translational axes for five-axis measuring system, the laser interferometer was used to detect the positioning errors in the closed-loop motion control mode. The positioning error curve of three motion axis was achieved by the approach of spline interpolation. In the meanwhile, the positioning errors were compensated by the way of modifying the indicating values of linear gratings. The VDI-3441 criterion was utilized to evaluate the positioning accuracy of such system before and after compensation. The experiment results illustrate the positioning accuracy has been significantly enhanced after compensation.
\end{abstract}

\section{Introduction}

At present, coordinate measuring machines are widely applied in many industrial areas, such as industrial inspection, machining, aerospace and national defense construction[1]. As a promising developing direction of three coordinate measuring machines, the related theories and technologies of multi-axis measurement system are paid more and more attention[2]. In general, the multi-axis measurement system consists of multiple translational and rotation axes. Restricted by many factors including the machining method and assembly process of parts, each motion axis contains six geometric errors[3]. Being the impact result of a number of geometric errors, the positioning error is a main error source that has a direct influence on the whole measuring accuracy of measurement system. For the open-loop control system, positioning errors are mainly determined by the drive system. For the closed-loop control system, positioning errors mainly depend on drive system, servo system and detection system. Under the premise that the positioning error meets certain demand of repeat accuracy, it is convenient and feasible that error compensation techniques are employed to improve the positioning accuracy of system.

Aiming at the five-axis measurement system exploited, the laser interferometer is used to inspect the positioning errors of three translational axes respectively. The positioning error curve of each axis is achieved by the method of cubic spline interpolation. The positioning error compensation is realized by modifying the indicating values of gratings. Consequently, the measuring accuracy of whole system is significantly enhanced.

\section{System introduction}

As shown in Fig.1, the miniature five-axis measuring system consists of five motion control axes, namely X, Y, Z, A and C axis. The strokes of XYZ axes are $220 \mathrm{~mm} \times 160 \mathrm{~mm} \times 60 \mathrm{~mm}$ respectively. The ones of $A C$ axes are $\pm 105^{\circ}$ and $\pm 90^{\circ}$ separately. The resolutions of linear gratings attached on $\mathrm{XYZ}$ axes are all $0.05 \mu \mathrm{m}$, and the angle resolutions of AC axes gratings are both $2^{\prime \prime}$. The step motors with subdivision function are used to drive each motion axis. The laser probe located at the end of beam can rotate around $\mathrm{AC}$ axes while travelling along $\mathrm{Z}$ axis. The tested workpiece clamped by a special fixture is fixed on the worktable, which can move in XY plane. 


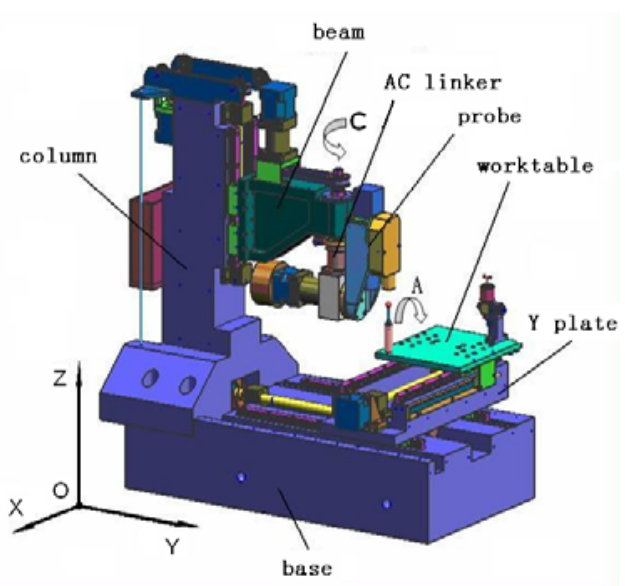

Fig.1 Five-axis measuring system

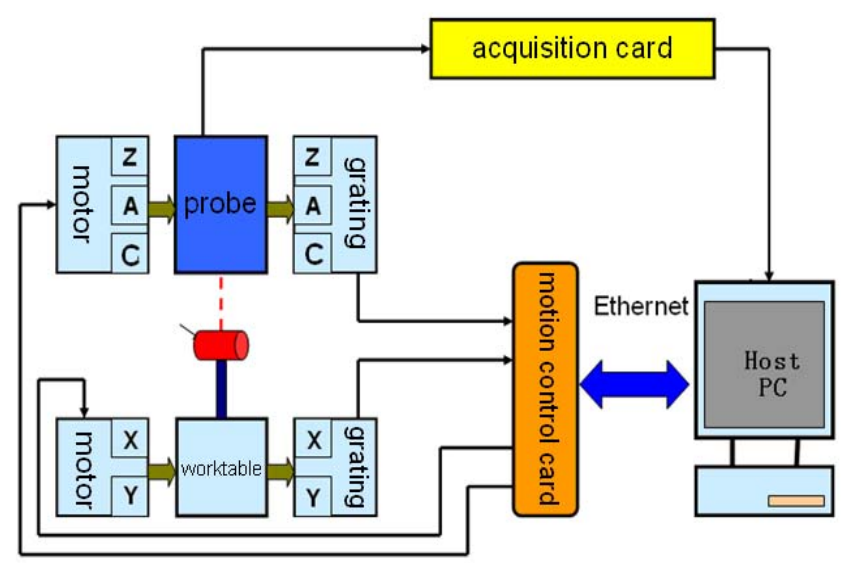

Fig.2 Working principle of this system

\section{The working principle of system}

Fig. 2 presents the working principle of system. The tested workpiece is fixed on the worktable and can travel along XY directions. The laser probe is located at the end of beam and has three motion freedoms: movement along $\mathrm{Z}$ axis, rotation around $\mathrm{A}$ axis and rotation around $\mathrm{C}$ axis. The industrial host computer communicates with the motion control card in accordance with TCP/IP protocol. The control motion card receives the instructions from the host computer while reading the position information of each motion. Therefore, the drive system and detection system compose of the closed-loop motion control system. The surface point cloud of tested workpiece is obtained by moving the worktable and adjusting the posture of laser probe. Consequently, the 3D inspection of workpiece is realized by virtue of the measurement mathematical model.

\section{Positioning error detection and compensation}

The positioning error of measurement system is the one between the actual reached position and the ideal position of the worktable or probe. The positioning error is a main error source which influences the measurement accuracy of system. Generally, each motion axis contains six geometric errors. However, it is only need to consider the combined effect of various geometric errors when the positioning error of single axis is detected and compensated. The positioning error can be expressed as formula 1.

$$
E_{i}=L_{i}-G_{i}
$$

Here, $L_{\mathrm{i}}$ says the actual position of motion part, which is usually substituted by the testing result of laser interferometer. $G_{\mathrm{i}}$ is the indicating value of gratings. The error compensation principle lies in modifying the indicating values of gratings with positioning error $E_{\mathrm{i}}$.

$$
L_{i}=G_{i}+E_{i}
$$

Usually, relative to the reference zero position of each axis, a series of positioning error values can be achieved at certain displacement interval. In order to calibrate the values of gratings continuously, cubic spline interpolation method is employed to obtain the error value of each axis in arbitrary position.

\section{Experiments}

The error inspection schemes of $\mathrm{XYZ}$ axes are similar. Taking $\mathrm{X}$ axis for example, the experiment device is shown in Fig. 3 which contains the laser interferometer, a tripod, a reference mirror, a target mirror, a specific fixture and an industrial host computer. The adopted laser interferometer named XL-80 comes from Renishaw corporation, its main working parameters are listed in table 1. 
Table 1 Working parameters of XL-80

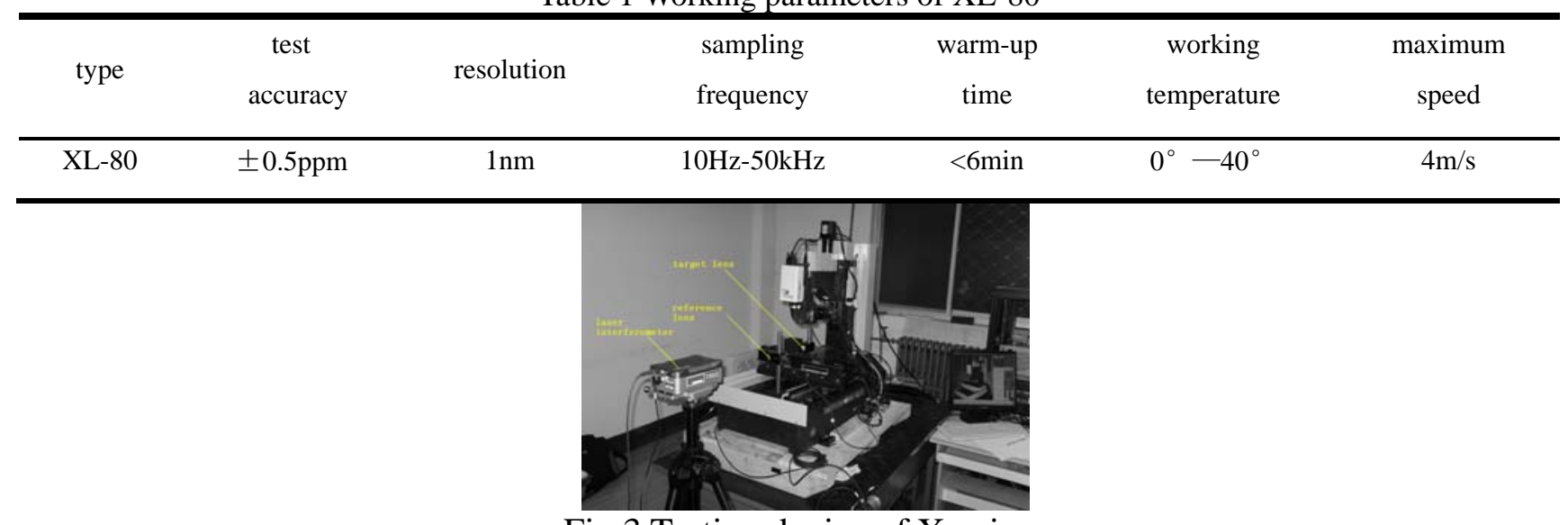

Fig.3 Testing device of $\mathrm{X}$ axis

The stroke of $X$ axis is $220 \mathrm{~mm}$. The sampling step is set to be $10 \mathrm{~mm}$. Fig.4 presents the positioning error curves with repeated five times. Fig.5 exhibits the mean positioning error curve for five testing results. Fig. 6 shows the error interpolation curve. After compensation, the fresh five testing error curves are displayed in Fig.7. By means of VDI-3441 criterion, the positioning error is improved from $6.12 \mu \mathrm{m}$ to 1.94

$\mu \mathrm{m}$.

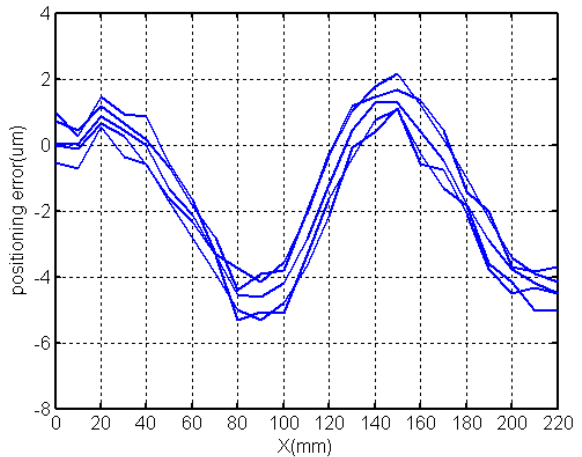

Fig.4 Positioning error curve of $\mathrm{X}$ axis

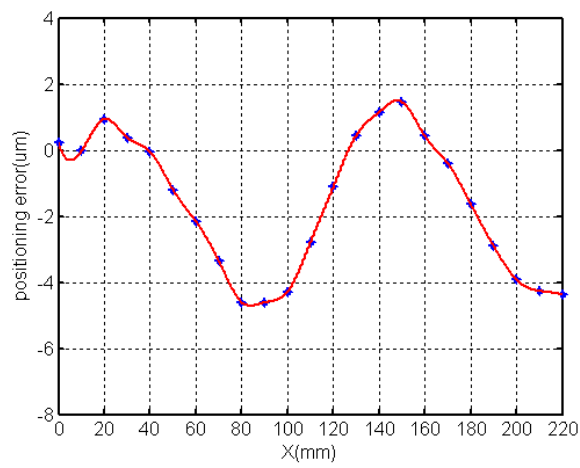

Fig.6 Positioning error interpolation curve of $\mathrm{X}$ axis

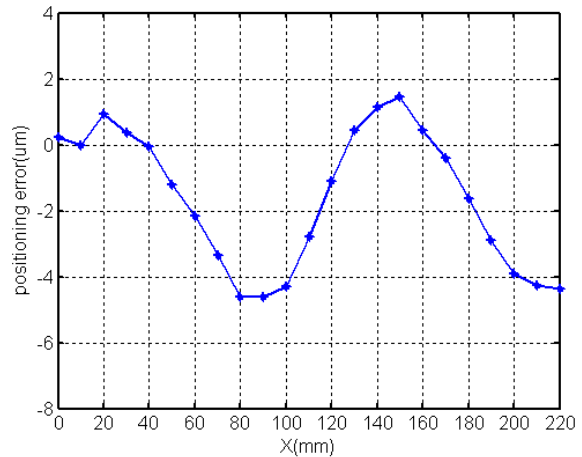

Fig.5 Mean positioning error curve of $\mathrm{X}$ axis

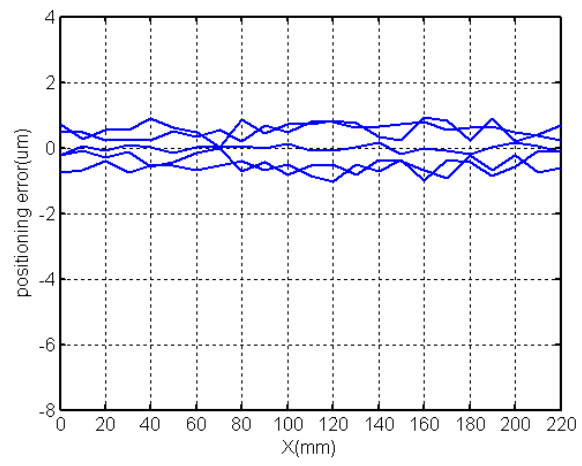

Fig.7 Positioning error curve of $\mathrm{X}$ axis after compensation

\section{Conclusion}

From above experiment results, it can be seen that the compensation method of positioning error introduced in this work is valid and feasible. Furthermore, it is no need to change the hardware configure of this five-axis measuring system. The positioning accuracy is greatly enhanced after compensation.

\section{Acknowledgements}

We take the opportunity to express our gratitude to Zhengzhou Science and Technology Bureau 
and Doctor Fund of Zhengzhou University of Light Industry, which provide sufficient financial support.

\section{References}

[1] Zuoshi Liu, Xiaojuan Ni. The status and development trend of CMM. Machinery, 2004, 42(480): 32-34.

[2] Guoxiong Zhang. Development orientations of coordinate measuring techniques. Infrared and Laser Engineering, 2008, 37(4): 1-5.

[3] Guoxiong Zhang. Three coordinate measuring machine. Tianjin University press, 1999.

[4] Yi Wang, Changjie Liu, Yongjie Ren, Shenghua Ye. Compensation for positioning error of industrial coordinate measurement robot. Journal of Mechanical Engineering, 2011, 47(15): 31-36. 